\title{
¿Qué es una relacion social ? Un conjunto de mediaciones diádicas
}

\author{
Michel Grossetti, CNRS y Université de Toulouse ${ }^{1}$
}

\begin{abstract}
Resumen
Los analistas de redes han desarrollado sofisticados métodos de recogida de información y de análisis de las estructuras de relaciones, pero raramente se han interesado en la definición de las relaciones en ellas mismas. El objetivo de este artículo es proponer un marco teórico que permita definir las relaciones interpersonales y precisar su lugar dentro del conjunto de formas sociales. La reflexión está basada en un estudio empírico sobre las redes personales realizado en el sur de Francia.
\end{abstract}

Palabras clave: mediación, relación, encaste, desacoplamiento.

\section{Résumé}

Les analystes de réseaux ont développé des méthodes sophistiquées de collecte d'informations et d'analyse des structures relationnelles, mais ne se sont que rarement intéressés à la définition des relations elles-mêmes. L'objectif de cet article est de proposer un cadre théorique permettant de définir les relations interpersonnelles et de préciser leur place dans l'ensemble des formes sociales. Le raisonnement est appuyé sur une étude empirique de réseaux personnels réalisée dans le sud de la France.

Mots-clés : mediation, relation, encastrement, découplage.

\begin{abstract}
Networks analysts have developed sophisticated methods of collecting and analysing relational data, but they have been rarely interested in the definition of the relations themselves. The aim of this article is to propose a theoretical framework allowing to define interpersonal relations, and to precise their place in the whole set of social forms. The reasoning is supported by a an empirical study of personal networks in the south of France.
\end{abstract}

Key words: mediation, relation, embedding, decoupling.

La mayoría de autores que trabaja el tema de las redes sociales estará de acuerdo en definir una red como un conjunto de relaciones, que no implica en ella misma ni una conciencia organizadora, ni un sentimiento de pertenencia, ni una frontera. Las cosas se complican un poco con los componentes fundamentales de la red, que son las relaciones sociales. En efecto, salvo algunas excepciones, la noción no ha sido

\footnotetext{
${ }^{1}$ Enviar correspondencia a: Michel.Grossetti@univ-tlse2.fr
} 
definida más allá del criterio empírico de la observación de un tipo particular de lazo. No obstante, una relación social no es sólo una construcción metodológica, ella es además una realidad compleja vivida y percibida por los actores sociales. De modo que es necesario dar un estatus teórico a esta noción. Aquello implica disponer además, en un plano anterior, de una concepción de conjunto del mundo social y del lugar en él que ocupan las relaciones y las redes. Se necesita entonces de una teoría general.

Tal concepción no podrá reducirse al postulado metodológico de « partir de las relaciones», que ha sido a menudo el credo común de los analistas de redes. Este credo ha sido perfectamente expresado en la introducción de una publicación colectiva que puede considerarse como un manifiesto del análisis de redes como paradigma sociológico (llamado « análisis estructural » en el libro). En dicha publicación, Wellman y Berkowitz afirmaban: «Invirtiendo la lógica tradicional de la encuesta en sociología, el análisis estructural considera que las categorías sociales (e.g. clases, razas) y los colectivos delimitados pueden ser puestos en evidencia y analizados de manera más adecuada al examinar las relaciones entre los actores sociales. En vez de comenzar por una clasificación a priori del mundo observable en un conjunto discreto de categorías, ellos comienzan por un conjunto de relaciones, a partir del cual construyen los planos y las tipologías de las estructuras sociales. » (Wellman y Berkowitz, 1988, p.3). Si esta opción metodológica, - partir de las relaciones - ha producido resultados de una gran fecundidad, ella se ha vuelto también, a menudo, una especie de reduccionismo en el análisis de las redes sociales. Las relaciones son naturalizadas y los actores olvidan su complejidad y su evolución, justamente aquello que ha sido criticado por uno de los fundadores de esta corriente, Harrison White (1995): «Es necesario notar (...) las dificultades que presenta la noción de lazo o de relación, sobre la cual se apoya ingenuamente una gran parte del aparato de análisis de las redes polivalentes. Una ambigüedad tal no es verdaderamente sorprendente: los lazos comienzan a resistir al análisis porque ellos devienen axiomáticos. » (White, 1995, p.712). Al centrarse en las relaciones, los analistas de las redes sociales tienden a dejar de lado los otros tipos de componentes de las estructuras sociales: las familias, las organizaciones, los colectivos, las comunidades son reducidas a conjuntos de relaciones, definidas ellas de una manera bastante sumaria. Todo esto puede conducir a una visión bastante pobre del mundo social, bajo la forma de un esquema de redes cuyas propiedades estructurales explicarían todos los fenómenos en los cuales se interesan los investigadores en ciencias sociales. 
Entre los investigadores que practican el análisis de las redes, varios han buscado dejar atrás esta definición un poco sumaria. Entre ellos, figura especialmente Harrison White $(1992,2008)$, para quien las relaciones no constituyen sino un elemento dentro de un vasto conjunto que él llama a veces "los contextos" (y que a menudo no nombra explícitamente), y que yo nombraré las "formas sociales". Estas formas ( « netdoms », « disciplinas », « estilos », « regimenes », « instituciones », etc.) son construcciones teóricas originales que se sustituyen a los colectivos, espacios sociales o a otros campos de la literatura sociológica. En este conjunto, las relaciones diádicas son construcciones analíticas extraídas por el investigador de un conjunto de narraciones producidas en permanencia por los actores: « Cada vínculo que persiste resume las luchas por el control. Cada vínculo es un equilibrio metastable entre las tentativas rivales de control, e induce así a descripciones regulares. Los vínculos describen las conexiones, pero no se trata de interconexiones eternas entre entidades fijas. Los vínculos reflejan siempre la actividad tal como es percibida por los observadores y los participantes, pero están también implicados en esta actividad, como pueden constatarlo tanto los observadores como los participantes. La acumulación de las descripciones, y también su evocación en otros vínculos, las estructura en modelos que tienden a ser percibidos como relatos.» (White, 2008, capítulo 2). Al poner el énfasis en la dimensión discursiva o narrativa de la vida social, la concepción de White rechaza los efectos de naturalización de los vínculos a los que podría conducir el análisis de las redes y abre así algunas perspectivas estimulantes. Pero su definición de las relaciones es demasiado ambigua y demasiado dependiente de su propia teoría para constituir una herramienta lo suficientemente robusta. Alexis Ferrand, otro analista de las redes, ha propuesto recientemente, desde una perspectiva sociológica más clásica, una definición de las relaciones basada en el tipo de regulación que se opera. Ferrand define tres tipos de regulaciones según ellas sean efectuadas a partir: de roles y normas (lo que él llama «regulación categorial»); de una posición en la estructura del conjunto de las redes («regulación reticular»); o de la confianza recíproca fundada en las interacciones pasadas entre los dos compañeros («regulación diádica») (Ferrand, 2007). Esta definición es muy clara y constituye un buen marco de referencia, pero ella incluye dentro de las relaciones interacciones efímeras como aquellas que se producen frecuentemente en los intercambios comerciales, por ejemplo, así como las interdependencias que resultan de la posición en las redes de dos actores sin contacto directo entre ellos. En los dos casos, se va más allá de aquello que los analistas de las redes consideran implícitamente o explícitamente como las relaciones. 
Los dos ejemplos muestran que no es fácil definir las relaciones sociales de una manera rigurosa, manteniéndose cerca de los trabajos habituales sobre las redes. El objetivo de este artículo es proponer una concepción de las relaciones y de las redes sociales que sea en lo posible compatible con los trabajos existentes, y que a la vez permita superar el reduccionismo relacional que las suele caracterizar. Para esto, este artículo se apoya por un lado en estudios empíricos de las redes personales, como la de una población de habitantes de la región de Toulouse que retoma un método clásico, y de otro lado en una teoría que hace de las redes un tipo de forma social entre otras (Grossetti, 2004), y moviliza ciertas ideas basadas en trabajos conducidos bajo la línea de lo que se ha comúnmente denominado la « teoría del actor-red ». En una primera parte, argumentaré a partir de resultados empíricos por la necesidad de no reducir el mundo social a una red, y de comprender cómo las relaciones interpersonales se articulan con compromisos dentro de formas colectivas de diferente naturaleza. Luego discutiré desde un punto de vista teórico esas formas colectivas, resaltando la noción de recurso de mediación para designar lo que permite a los actores de coordinarse en una organización, o a un nivel más general en un colectivo, sin apoyarse únicamente en relaciones personales. Mostraré que la articulación entre colectivos y relaciones personales puede ser muy bien comprendida a partir de las nociones de encastre y de desacoplamiento, parcialmente reformuladas a partir de la versión que Harrison White ha propuesto. Finalmente, concluiré con una definición de las relaciones sociales.

\section{1. ¿Cómo se construyen las relaciones personales?}

Para comprender las relaciones que constituyen las redes es necesario alejarse del reduccionismo relacional que hace de las relaciones el único componente de la estructura social y descuida a su vez definirlas. Una buena forma de convencerse que el mundo social no se reduce a una red es preguntarse por el origen de las relaciones sociales. Partamos de ellas cómo nos lo sugiere el análisis estructural, pero en lugar de preguntarnos qué es lo que ellas producen, tratemos de comprender de dónde vienen.

Claude S. Fischer, quién dirigió una investigación empírica sumamente importante sobre las redes personales hacia fines de los años 1970, resumía así sus conclusiones : « La mayoría de los adultos conocen a otras personas a través de su familia, en el trabajo, en el barrio, en las organizaciones, o por intermedio de sus amigos o de sus padres; ellos continúan frecuentando a las personas que conocieron en las situaciones citadas anteriormente, por ejemplo en la escuela o el 
ejército; es raro que los encuentros pasajeros, en un bar, en un centro comercial u otro lugar similar, se conviertan en algo más que simplemente encuentros breves » (Fischer, 1982, p.4). Dicho de otra manera, una vez que uno comienza a preguntarse por el origen de las relaciones individuales, se encuentra con el entorno colectivo (organizaciones, familias, etc.), al seno del cual estas relaciones se forman muy a menudo antes de lograr su autonomía. E. Goffman afirma algo similar: «La mayoría de las relaciones ancladas nacen, me parece, por razones que les son exteriores y son el resultado directo e inmediato de disposiciones institucionales, (se puede citar como ejemplo los hermanos y las hermanas, los clientes, los colegas de trabajo, los vecinos) (...)Desde luego, esos contactos [en el curso de los cuales se afirman las relaciones] reenvían ellos mismos a las organizaciones sociales, que constituyen un marco extenso y una ocasión: vecindad, escuelas y universidades, lugares de trabajo, recepciones, temporadas de verano y otros » (Goffman, 1977, pp.136-137). Recientemente, en su bello trabajo sobre la amistad, Claire Bidart va en el mismo sentido: « No se encuentra amigos en la calle, en la muchedumbre, a partir de la nada. Ciertos marcos, ciertos lugares, ciertos medios son relativamente favorables a la construcción de vínculos interpersonales, mientras que otros lo hacen muy difícil » (Bidart, 1997, p.52).

Por mi parte, dirigí el 2001 una investigación que transpuso el método utilizado en 1977 par Claude Fischer en la región de San Francisco (Fischer, 1982). Esta investigación fue realizada en la región de Toulouse y en una zona rural situada a una hora de Toulouse (Grossetti, 2005, 2007). En ella se utiliza una serie de generadores de nombres que permiten constituir una lista de relaciones (27 en promedio en la investigación de Toulouse) de la cual se extrae una sub-muestra de 5 relaciones máximas para las preguntas complementarias. 399 personas fueron interrogadas (300 en la aglomeración de Toulouse y 99 en un cantón rural de Tarn). Ellas citaron 10932 personas de las cuales 1624 respondieron a preguntas complementarias, de entre las cuales una se refería a los contextos iniciales de encuentro con las personas citadas. En el caso de 1606 de entre ellas, el origen del encuentro fue mencionado con suma precisión por el entrevistado. La Tabla 1 presenta una categorización de las respuestas. 


\begin{tabular}{|c|c|c|}
\hline Contexto de encuentro & $\mathbf{N}$ & $\%$ \\
\hline Familia & 487 & 30,3 \\
\hline Escuela & 79 & 4,9 \\
\hline Universidad & 79 & 4,9 \\
\hline Trabajo & 218 & 13,6 \\
\hline Asociaciones & 90 & 5,6 \\
\hline Organizaciones & 466 & 29 \\
\hline Total familia y organizaciones & 953 & 59,3 \\
\hline Vecinos & 123 & 7,7 \\
\hline Amigos de la infancia & 17 & 1,1 \\
\hline A través de los hijos & 110 & 6,8 \\
\hline A través de la pareja & 100 & 6,2 \\
\hline Por un amigo & 202 & 12,6 \\
\hline Total sociabilidad ("a través...") & 429 & 26,7 \\
\hline Otros (azar, etc.) & 101 & 6,3 \\
\hline Total & 1.606 & 100,0 \\
\hline
\end{tabular}

Tabla 1. Contextos de construcción de las relaciones sociales (Encuesta Toulouse, 2001)

Estas categorías y proporciones son desde luego dependientes del procedimiento de la investigación y de la selección de la sub-población para la cual esta pregunta fue planteada. Los resultados son bastante próximos de aquellos que obtuvo Fischer siguiendo el mismo procedimiento con una población de la región de San Francisco en los años 1970, lo que probablemente pueda concederle una generalización.

Lo que me interesa de estos resultados, es que aparecen, al origen de una gran parte de las relaciones sociales, contextos colectivos (familias y organizaciones) que no pueden reducirse a la figura de la red.

Vimos al comienzo que el postulado de los analistas de redes reposa en la idea de « partir de las relaciones ». Pero si uno parte de las relaciones para remontar al origen de la red, uno no se encuentra solamente con otras relaciones, sino que también, sobre todo, con formas sociales diferentes, que pueden difícilmente explicarse como simples superestructuras de las redes. Es necesario, entonces, realizar una breve examen teórico para definir con mayor precisión estas formas sociales, que son a la vez alternativas a las redes y que interactúan con ellas. Volveremos luego el tema de las relaciones sobre una base teórica más sólida. 


\section{Colectivos y mediaciones}

¿Qué es lo que diferencia una red de una organización o de una familia, dos formas sociales que yo incluiré aquí dentro de la noción general de « colectivo »? Esta diferencia se percibe muy bien en el trabajo del sociólogo de ciencias Nicholas Mullins, uno de los pocos autores que ha conceptualizado el paso de uno de esos tipos de forma social al otro, en un estudio sobre la emergencia de las especialidades científicas: «Un colectivo se forma cuando los investigadores se vuelven concientes de sus estructuras de comunicación y comienzan a trazar fronteras en torno de aquellos que trabajan en su mismo tema. Se desarrolla por la recombinación de pares y triadas en respuesta a condiciones favorables, e.g.: la oportunidad, el liderazgo, un problema substancial de investigación, una o varias instituciones de apoyo. Esos colectivos son a menudo identificados por un nombre, tanto por aquellos que son al interior como por aquellos que son al exterior, son más estables que los pares o triadas que los constituyen, tienen una cultura específica y son capaces de obtener los medios y los estudiantes.» (Mullins, 1972, p.69) . El colectivo de Mullins emerge de una red pre-existente, que él contribuye a reconfigurar. Él presenta algunos ingredientes que no son necesarios para la existencia de una red: fronteras, un nombre, una cultura específica, un relato sobre la historia del colectivo en cuestión, criterios más o menos explícitos de pertenencia, recursos más o menos compartidos. ¿Cómo calificar estos ingredientes? Lo más simple seria considerarlos como recursos, algunos de los cuales permiten a los miembros del colectivo de coordinarse sin necesariamente apoyarse en relaciones interpersonales. Aquí podemos retomar una idea propuesta por Antoine Hennion, un autor cercano en ciertos aspectos de los estudios conocidos bajo el nombre de «teoría del actor- red». Él ha desarrollado la noción de «mediación» para designar a todos los actores sociales, les dispositivos técnicos o los objetos ordinarios que crean un vínculo entre los actores. Al estudiar el renacimiento de la música barroca en los treinta últimos años, Hennion mostraba que no se podía comprender lo ocurrido sin tener en cuenta las partituras, a los musicólogos y sus obras, los propios instrumentos. Llamemos «recursos de mediación» a todos estos elementos que vinculan a los actores entre ellos sin llegar a ser por ello relaciones sociales en el sentido que le dan los analistas de las redes al término y que busco precisar en este texto. Un colectivo se define entonces por el acceso de sus miembros a los recursos comunes, algunos de los cuales operan como mediadores entre sus miembros. Evidentemente, los miembros del colectivo no poseen igualmente acceso a los diferentes recursos, pero su calidad de miembro les da al menos una posibilidad de acceso que no existe para los actores exteriores 
al colectivo. Es también evidente, que las fronteras del colectivo no son claramente definidas y pueden ser el objeto de luchas por su definición, de diferentes tipos de transgresiones. Ellas constituyen en definitiva un recurso como cualquier otro.

Para precisar esta idea, podemos tomar también el célebre ejemplo del estudio del acceso al empleo realizado por Granovetter (1974). En este estudio, si el 56\% de los empleos habían sido encontrados por intermedio de relaciones sociales, el $44 \%$ fueron obtenidos por otros medios: candidaturas espontáneas, respuestas a anuncios, por intermedio de agencias de empleo, etc. Enviar una candidatura espontánea supone disponer de información sobre una empresa (dirección, actividad, etc.). Si esas informaciones no se obtienen a través de las relaciones, ellas pueden conseguirse a través de la consulta de anuarios o de la prensa económica, por ejemplo. Esos son dos ejemplos de recursos de mediación que estructuran el mercado de trabajo. De la misma manera, una agencia de empleo constituye un recurso de mediación. En todos los casos, estos recursos han sido concebidos y dispuestos para producir mediaciones, ayudar a las personas que buscan empleo y ofrecerles un primer contacto con el empleador. Nosotros podríamos utilizar la expresión « dispositivo de mediación » para designar un recurso o un conjunto de recursos concebidos para operar como mediadores. Si he privilegiado el término « recurso », se debe a que las mediaciones pueden ser operadas a partir de lugares, objetos o personas cuyo rol no es explícitamente ése. Basta con pensar en el caso de los vendedores ambulantes, para quienes la mediación con los clientes está constituida por el lugar en él mismo (uno sabe que tiene la posibilidad de encontrar a tal de vendedor en tal calle o tal plaza).

El ejemplo de los anuncios en los diarios o de las agencias de empleo muestra que los recursos de mediación no se limitan a los colectivos explícitamente reconocidos como tales por sus miembros, como lo pueden ser las organizaciones o las familias. Ellos pueden también estructurar colectivos con contornos más laxos, que no generan necesariamente un sentimiento de pertenencia, como los mercados, por ejemplo (el mercado de trabajo analizado por Granovetter). Tomemos el ejemplo de un recurso de mediación clásica, un periódico. Aquellos que leen el periódico pueden tener la sensación de pertenecer a una comunidad de lectores, sobre todo si lo leen regularmente, pero pueden considerarlo también como una simple fuente de información totalmente substituible por otra. Es posible circunscribir analíticamente un colectivo a partir de los lectores del periódico - es, de hecho, lo que hacen los directores de periódicos y los periodistas apoyándose en las estadísticas de venta y los estudios de mercado-, pero ese colectivo tiene fronteras 
imprecisas y una identidad fluctuante. Para ciertos periódicos militantes, el colectivo de lectores presenta una estabilidad y una identidad fuertes, que puede acercarse más a ser una organización, mientras que para la mayor parte de periódicos ese colectivo es más fluctuante y asocia un núcleo de lectores regulares a los lectores ocasionales, atraídos puntualmente por un titular o una información.

Los colectivos son, entonces, formas sociales específicas caracterizadas por la puesta en común, entre los actores, de ciertos recursos, algunos de los cuales son recursos de mediación que les permite coordinarse sin apoyarse integralmente en las relaciones interpersonales. Hemos visto en la primera parte que estos colectivos, y los recursos de mediación que le son específicos, están a menudo al origen de las relaciones interpersonales. El estudio de Mullins muestra también que los colectivos pueden construirse a partir de las redes de relaciones diádicas. Es necesario, entonces, rendir cuenta de las interacciones entre los colectivos, las relaciones y las redes. Para ser más preciso, es necesario inscribir esas interacciones en una dinámica global de emergencia o de cambio de diferentes formas sociales. Para eso, es necesario salir de una concepción estática del mundo social, en la cual las cosas existen o no existen, para adoptar más bien una concepción más dinámica, en la cual ellas emergen o se disuelven.

\section{Encastre y desacoplamiento}

Para analizar esta dinámica de las formas sociales, se puede utilizar las nociones de encastre y desacoplamiento que he debido en parte redefinir (Grossetti, 2004) partiendo del sentido que les dio Harrison White (1992, 2002). En el presente texto, el encastre es el incremento de las dependencias de una forma social con respecto a otra y el desacoplamiento es el proceso recíproco de autonomización. Cuando un colectivo emerge con respecto a la red que le ha dado origen, él se desacopla: mientras que al comienzo su funcionamiento es totalmente dependiente de la red, de su estructura y de sus actores centrales, gracias a los recursos de mediación él adquiere una relativa autonomía, lo que puede evaluarse en su capacidad para sobrevivir al alejamiento de ciertos actores centrales. Al mismo tiempo, se integra en una red compuesta por otros colectivos del mismo tipo, es decir, se reencastra, pero a un nivel diferente. Los procesos de encastre y de desacoplamiento a diferentes niveles están en tensión permanente, pero pueden estabilizarse momentáneamente en un nivel dado de dependencia entre las entidades implicadas. Para una organización, este equilibrio puede establecerse en un nivel muy alto de dependencia en la relación de los individuos y sus relaciones, a tal punto que el alejamiento de un solo actor o la ruptura de una sola relación 
puede llevar a una importante reconfiguración del colectivo o incluso a su desaparición. Inclusive, la organización puede haber creado recursos de mediación tan fuertes que ella se muestra insensible a los cambios de sus miembros y de sus relaciones, de manera que ellos parecen totalmente sustituibles. En realidad, las organizaciones pueden encontrarse en todas las situaciones intermedias y pasar de una a otra en el curso del tiempo. A niveles de acción más amplios, estas mismas nociones funcionan muy bien. En el fondo, la emergencia de la economía de mercado como esfera relativamente autónoma corresponde a la constitución de dispositivos jurídicos y materiales que son a su vez fuentes de desacoplamiento (Polanyi, 1983). A los niveles intermedios, constituidos por los mercados particulares, se puede medir el grado de autonomización de los intercambios en relación a las relaciones personales y a otros mercados o esferas de actividad. Así, si en el mercado de trabajo en Francia, entre 30 y $40 \%$ de los empleos son encontrados por relaciones (Forsé, 1997), aquello significa que el desacoplamiento de ese mercado, que pasa por todas las mediaciones que cité anteriormente así como por otras más específicas (en Francia, por los concursos de la función pública, por ejemplo), se ha estabilizado a un nivel limitado de autonomía frente a las relaciones personales. Por otro lado, podemos suponer que el número de compras en el supermercado efectuadas en base a las relaciones personales con los vendedores es extremadamente débil, lo que autonomiza el mercado de la « gran distribución » frente a las redes sociales, pero lo hace también más dependiente de todos los dispositivos y profesionales del marketing, del packaging y de otras actividades de mediación (Cochoy, 2002).

El desacoplamiento de las organizaciones o de los colectivos implica la creación de recursos de mediación que permitan liberarse de las características de los actores individuales y de sus relaciones. Su encastre, en cambio, se mide en la ineficacia de estos recursos y en la importancia asumida por las redes interindividuales en su actividad. Hasta aquí hemos razonado « desde abajo hacia arriba » o « de lo micro hacia lo macro », es decir, a partir de los actores y de sus relaciones hacia las organizaciones y los mercados. Pero es posible invertir la perspectiva y preguntarse por el encastre y el desacoplamiento de las relaciones interpersonales en relación a su contexto de emergencia.

Podemos ahora volver al problema de la emergencia de las relaciones, apoyándonos en un cuadro teórico más sólido. 


\section{Tipología de los contextos de la emergencia de las relaciones}

Hemos visto anteriormente que desde que se aborda empíricamente la cuestión de la emergencia de las relaciones interpersonales, uno se encuentra con los colectivos: la familia, el organismo de trabajo, los grupos de diversión, etc.

Tomemos el ejemplo de una persona contratada en una empresa. La organización la lleva a forjar relaciones con otros miembros de la empresa. Supongamos ahora que la empresa esté quebrando. Los empleados buscarán trabajo en otra parte. Una buena parte de los débiles lazos que habían creado desaparecerán. Pero ciertos vínculos sobreviven a la desaparición del colectivo. Por razones muy diferentes (afinidad intelectual, proximidad social, compatibilidad en el entorno) ellos se refuerzan y no son más relaciones de trabajo sino vínculos que podrán ser calificados de amicales por los protagonistas. ¿Qué pasó? En un comienzo esas relaciones estaban enmarcadas fuertemente por la organización de la empresa, la división del trabajo, los protocolos. Luego, se fueron progresivamente desacoplando. Ese desacoplamiento no necesita de la desaparición del marco que constituye la empresa, desaparición que introduje aquí para contrastar y dramatizar un poco la situación. Él comienza cuando la relación supera los roles previstos por la organización, cuando se personaliza, cuando los protagonistas no son más sustituibles el uno por el otro. Este desacoplamiento está siempre en tensión con el encastre que es resultado de la disciplina de la empresa. Una relación creada en el marco de un colectivo se desarrolla siempre al margen de las reglas del colectivo y en contradicción parcial con su integridad, su identidad en tanto que colectivo.
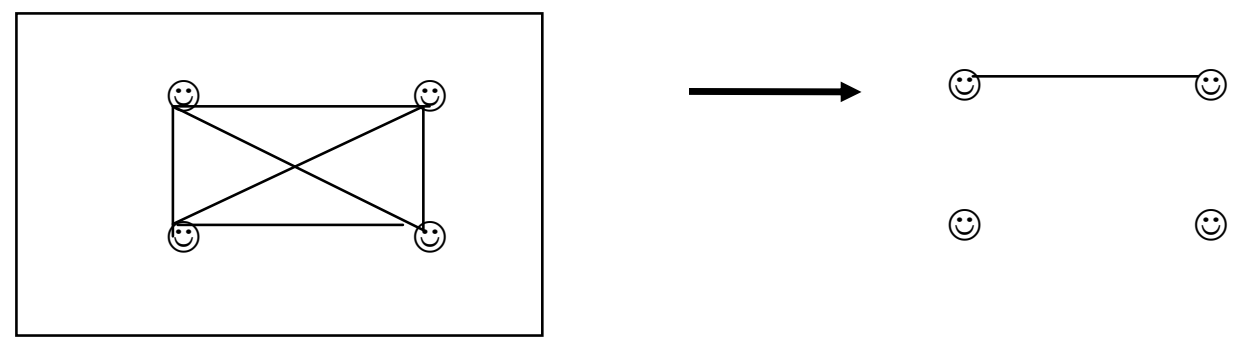

Figura 1. De los colectivos a las relaciones.

Las relaciones pueden entonces encastrarse en entidades más extensas, los colectivos. Ellas pueden también encastrarse en sus componentes, siguiendo las interacciones. El desacoplamiento de la relación es también la constitución de una historia compartida que permite a la relación de adquirir una consistencia que excede la simple adición de intercambios. El desacoplamiento tiene evidentemente 
algo que ver con la fuerza del vínculo: una de las características de un vínculo fuerte es el de ser poco sustituible.

Pero las relaciones no nacen solamente en los colectivos. Una parte importante de entre ellas nace de la existencia de otras relaciones: estar en relación con alguien es incrementar la probabilidad de entrar en contacto con aquellos con quienes él también está en relación. Por medio de las presentaciones, las recomendaciones o los padrinazgos, la red se completa, de alguna manera, por ella misma, sin necesariamente poner en juego los colectivos.
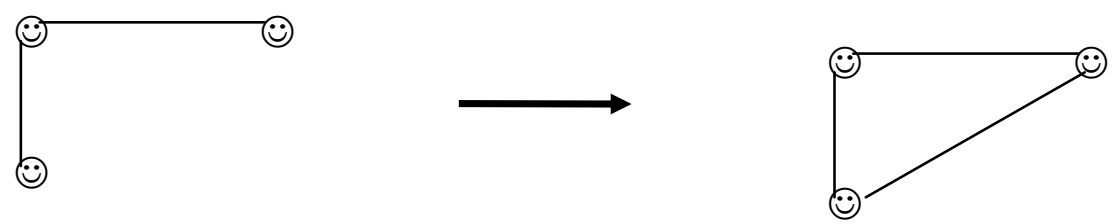

Figura 2. De las relaciones a las relaciones.

En el segundo caso de figura, se puede también ver la construcción de la tercera relación directa como un desacoplamiento en relación a los intercambios que pasaban anteriormente por intermedio del « amigo común », entonces, en relación a la red. El desacoplamiento puede medirse en la capacidad de los protagonistas de departir en la ausencia del intermediario, y por la capacidad de la relación de resistir a la desaparición del intermediario. Pero, al mismo tiempo que la relación aparece y se desacopla, ella toma su lugar en la red y se encastra.

Finalmente, existe un tercer tipo de contexto de creación de relaciones. Es el caso en que los dos protagonistas se aproximan debido su interés por un mismo recurso, por un mismo reto. El análisis de la constitución de la biología molecular realizado por Mullins, evocado antes, es de nuevo un buen ejemplo. ¿Por qué los investigadores comienzan a entrar en contacto los unos con los otros? Mullins evoca los investigadores «que pasaron de un punto de vista a otro, y que son o no en comunicación. (...) un conjunto de individuos tal que todos se encuentran en la misma situación cognitiva con respecto a un mismo problema o a problemas similares » (Mullins, 1972, p.55). Esos investigadores pueden acercarse por lecturas comunes (en este caso, un artículo fundador de Shrödinger). Los actores están vinculados aquí por un mismo recurso cognitivo, esto los acerca y lleva a algunos de entre ellos a tratar de conocer a los otros. Este ejemplo se generaliza 
fácilmente en todos los casos donde los investigadores entran en contacto durante un coloquio: si ellos van a participar en él es porque comparten intereses comunes. Esos encuentros son desde luego facilitados por los colectivos que en una cierta medida los enmarcan: la comunidad científica general en el caso de la biología molecular, tal colectivo disciplinario en un coloquio. Los encuentros son inducidos por la relación a un mismo recurso pero ellos se apoyan en otros recursos compartidos al seno del colectivo. La diferencia con el primer tipo de encuentro, directamente inducido por un colectivo, es que en esta situación los actores no están en contacto por su simple presencia en el colectivo. El colectivo es suficientemente amplio como para que, eventualmente, ellos no puedan jamás entrar en contacto si no comparten un mismo interés en un momento dado. El colectivo es entonces, en este caso, un simple marco. Se puede generalizar este ejemplo a los encuentros de los amantes de una actividad de ocio y a las relaciones con los comerciantes o con los profesionales próximos. Se puede también incluir en este tipo ciertos encuentros amorosos, ya sea resultado de situaciones de « conquista » en lugares anónimos, en lugares específicos (discotecas), o en momentos específicos (bailes). Finalmente, lo que está en juego aquí es la relación social en ella misma. Para acabar esta lista, que ciertamente no es exhaustiva, es necesario mencionar las relaciones con los vecinos, uno de cuyos aspectos es el compartir intereses comunes (una proximidad a saber cuidar, espacios públicos comunes, etc.).

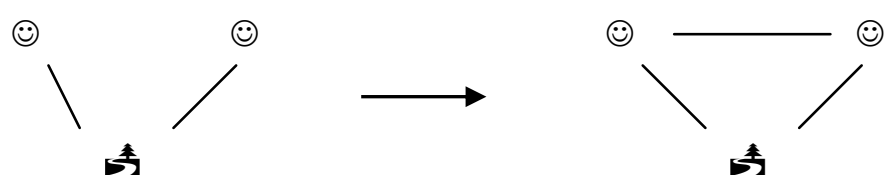

Figura 3. Retos comunes a las relaciones.

El desacoplamiento se produce aquí a diferentes niveles. Para empezar, está la relativa pérdida del objeto intermedio en beneficio del vínculo directo, como en el caso precedente. Está también, como en el primer tipo de contexto de encuentro, el desacoplamiento en relación a los colectivos en los cuales la relación está eventualmente inscrita. El desacoplamiento se mide por la capacidad de la relación a sobrevivir a la eventual desaparición de los elementos intermediarios o a la salida del colectivo. 
Sin embargo, las relaciones no se quedan necesariamente prisioneras de los contextos en los cuales se crean: ellas se desacoplan. En el estudio sobre las redes personales de los habitantes de la región de Toulouse, nosotros pedimos a los interrogados una calificación de las personas citadas siguiendo una serie de roles relacionales clásicos (familia, colegas, vecinos, miembros de una asociación, etc.). Cuando se compara esas calificaciones con las designaciones en los contextos de creación de las relaciones se observa muchos cambios. Ciertos contextos han desaparecido: es el caso, por ejemplo, de la escuela o de la universidad. Las personas que se encontraron en la universidad y que son citadas en este estudio se volvieron parejas (13\%), amigos (la mayoría de los otros), pero también algunos se hicieron colegas (38\%). Se puede percibir la amplitud de la evolución de las relaciones con respecto a un contexto de origen tomando el caso de las relaciones de trabajo (Tabla 2). He decidido hacer figurar en este cuadro la proporción de personas consideradas como próximas en cada categoría, a fin de mostrar la variabilidad en los tipos de relaciones nacidas en el marco del trabajo, según su evolución ulterior.

\begin{tabular}{|l|l|c|c|c|}
\hline $\begin{array}{c}\text { Contexto de } \\
\text { encuentro }\end{array}$ & Designación actual & Efectivo & \% & \% próximos \\
\hline Trabajo & Solo colega & 33 & $15 \%$ & 21 \\
\hline Trabajo & Colega y amigo/a & 66 & $29 \%$ & 64 \\
\hline Trabajo & Solo amigo/a & 82 & $36 \%$ & 48 \\
\hline Trabajo & Cónyuge o familia & 29 & $13 \%$ & 90 \\
\hline Trabajo & Otra designación & 15 & $7 \%$ & 0 \\
\hline \multicolumn{2}{|r|}{ Total trabajo } & $\mathbf{2 2 5}$ & $\mathbf{1 0 0 \%}$ & $\mathbf{4 8}$ \\
\hline Otro & Solo colega & 9 & $17 \%$ & 0 \\
\hline Otro & Colega + amigo & 44 & $83 \%$ & 52 \\
\hline \multicolumn{2}{|c|}{ Total colegas encontrados fuera del } \\
trabajo
\end{tabular}

Tabla 2. Antiguos y nuevos «colegas».

La calificación de «amigo» consigue de alguna manera atraer relaciones cuando aquéllas adquieren intensidad (es testimonio de ello el hecho de que las personas encuestadas designan a estas personas como próximas). La Tabla 3 conjuga los contextos de creación de relaciones con aquellos que son considerados como amigos al momento de la encuesta. 


\begin{tabular}{|c|c|c|c|}
\hline Contexto de encuentro & Amigos & $\%$ & $\begin{array}{c}\text { \% por el conjunto de las } \\
\text { relaciones (sin contar la } \\
\text { familia) }\end{array}$ \\
\hline Misma familia & 21 & $3 \%$ & \\
\hline Escuela & 53 & $7 \%$ & $7 \%$ \\
\hline Crianza conjunta & 14 & $2 \%$ & $\mathbf{2} \%$ \\
\hline Estudios superiors & $\mathbf{1 1}$ & $\mathbf{1 2} \%$ & $\mathbf{9 \%}$ \\
\hline Trabajo & 62 & $9 \%$ & $7 \%$ \\
\hline Asociaciones & 155 & $22 \%$ & $19 \%$ \\
\hline 2. Estudios sup. / trabajo / & 62 & $9 \%$ & $\mathbf{3 4 \%}$ \\
\hline assoc. & $\mathbf{2 9}$ & $\mathbf{4 0 \%}$ & $\mathbf{1 1 \%}$ \\
\hline $\mathbf{3 .}$ Vecinos & $\mathbf{4 6}$ & $\mathbf{6 \%}$ & $10 \%$ \\
\hline Por les hijos & 55 & $8 \%$ & $9 \%$ \\
\hline Por el cónyuge & 51 & $7 \%$ & $18 \%$ \\
\hline Por otro amigo & 140 & $19 \%$ & $\mathbf{3 7 \%}$ \\
\hline $\mathbf{4 . ~ S o c i a b i l i d a d ~ ( ~ “ p o r . . . " ) ~}$ & $\mathbf{2 1}$ & $\mathbf{3 4 \%}$ & $\mathbf{9 \%}$ \\
\hline $\mathbf{5 . ~ O t r o ~ ( a z a r , ~ e t c . ) ~}$ & $\mathbf{6 2}$ & $\mathbf{9 \%}$ & $\mathbf{1 0 0 \%}$ \\
\hline Total & $\mathbf{7 1 9}$ & $\mathbf{1 0 0}$ & \\
\hline
\end{tabular}

Tabla 3. Contextos de encuentro de los «amigos».

Se observa que los amigos se crean en los mismos contextos que las relaciones consideradas de manera más general, de modo tal que la familia juega evidentemente un rol muy restringido (los « amigos » encontrados por intermedio de la familia son casi siempre primos lejanos). El pasar de un contexto preciso (un colectivo como aquellos de los estudios o del trabajo, una relación intermediaria o un reto común) a una designación como la de « amigo » es un índice del desacoplamiento de las relaciones concernidas. ¿Cómo se opera ese desacoplamiento?

Preguntarse por el desacoplamiento de las relaciones, significa interrogarse por aquello que las constituye y que se aleja de aquello que heredan de su contexto de origen. En el caso de los colectivos, hemos visto que el desacoplamiento implica la creación de recursos de mediación. Debe ocurrir entonces lo mismo con las relaciones. ¿Cómo son estos recursos de mediación específicos a las relaciones?

\section{5. ¿Qué es una relación interpersonal?}

Podemos ahora tratar de definir las relaciones interpersonales.

Afirmé antes que el desacoplamiento de una relación a partir de un contexto de trabajo comienza cuando la relación supera los roles previstos para la organización, cuando los protagonistas no son más sustituibles uno por el otro. ¿Qué hace que dos personas no sean más substituibles la una por la otra? La primera respuesta que viene lógicamente a la mente es que al término de las interacciones cada una 
de ellas adquiere sobre la otra una serie de informaciones que ella toma en cuenta en su propio comportamiento. Podemos llamar a eso un conocimiento recíproco. Este conocimiento produce un ajuste entre los compañeros.

Se pueden definir bien las relaciones interpersonales únicamente a partir del conocimiento recíproco de los pares. Pero es fácil de notar que esto no es consigue corresponderse con el sentido habitual que se le da a estas relaciones en el análisis de las redes sociales. Para empezar, el conocimiento recíproco puede construirse sin interacción directa entre los pares, las informaciones pueden pasar a través de intermediarios (boca a boca) o a través de los recursos genéricos de mediación (los medias). Pero, incluso si se uno se impone pensar que este conocimiento se haya construido en las interacciones, esto no llega a ser suficiente. En efecto, un conocimiento como éste puede muy bien construirse entre adversarios, sea en un deporte o en un conflicto. Sin embargo, uno de los presupuestos implícitos en la mayoría de los análisis de redes es que las relaciones son lo suficientemente no conflictivas como para que los recursos puedan circular. Bien entendido, los recursos circulan aún cuando existe un conflicto o una adversidad entre los pares. Por ejemplo, cuando en un conflicto uno de los protagonistas ejecuta una nueva táctica, da una información que su adversario puede decriptar, de modo que él puede también tratar de adoptar esta táctica. No obstante, en los análisis de las redes suponemos en general que, incluso si las personas en relación son rivales, ellas están también comprometidas en una suerte de cooperación, al menos mínima. Para especificar esto, la noción de compromiso nos puede ser útil. Estar comprometido en una relación social con una persona es dar por sentado que existe una cooperación, así sea mínima. Se puede, por supuesto, estar comprometido de manera negativa en una relación antagónica con alguien. En lo que sigue, trataré solamente el compromiso « positivo », que implica una cooperación.

El grado mínimo de compromiso relacional es reconocer que se está en relación con alguien. Basta imaginar una persona que se encuentra con otra en una fiesta y se dirige a ella evocando su relación común. La persona interpelada puede ignorar esa evocación y comportarse como si aquella persona que se dirige a ella le fuera desconocida. Ella manifiesta así su rechazo a reconocer que existe un compromiso frente a la otra persona. A la inversa, si ella decide manifestar su compromiso, los otros participantes descubrirán inmediatamente la existencia de una relación a partir de las actitudes de los protagonistas y la manera en como se dirigen el uno al otro. La relación social entre dos personas puede entonces ser definida como « un conocimiento y un compromiso recíprocos fundados sobre interacciones». La noción 
de compromiso permite pasar fácilmente a las relaciones con las organizaciones o los colectivos ya que el compromiso puede tomarse de una forma contractual.

Se puede considerar el conocimiento y el compromiso como recursos de mediación que tienen por efecto encuadrar las interacciones de manera específica. Interactuar con una persona con la cual se tiene una relación supone implícitos y referencias que son más específicas que aquellas que resultan de la inscripción en un colectivo o en un contexto más amplio. El conocimiento y el compromiso producen otro recurso de mediación, que se asocia casi siempre a la noción de relación social, la confianza (Cook, 2001). Es una noción bastante compleja. La confianza que se tiene en una persona no funciona de manera binaria (si o no): se puede tener confianza en la actitud de la otra persona (su benevolencia con respecto a nosotros o su honestidad) pero no en su capacidad para resolver un problema, o a la inversa. La confianza puede difractarse en una miríada de aspectos de los cuales algunos serán implícitos y otros supondrán una reflexión. Lo que es importante aquí es que toda relación entre personas se acompaña de la existencia de una cierta confianza entre esas personas. Esta confianza puede añadirse o substituirse a aquella que engendran los dispositivos colectivos, pero ellas son bastante distintas. Las mediaciones específicas de las relaciones interpersonales no se extienden más allá de los compañeros de la relación. Llamémoslas entonces mediaciones diádicas. Podemos completar nuestra definición de la relación interpersonal: «Un conocimiento y un compromiso recíproco fundados en las interacciones que dan lugar a formas específicas de confianza entre los compañeros».

\section{Conclusión: formas sociales y área de eficiencia de las mediaciones}

Los actores se coordinan a través de recursos de mediación. Esos recursos son muy diversos. Ellos pueden ser de naturaleza cognitiva (lenguaje, información, normas culturales, roles sociales), materiales (medios de comunicación, medias, marcas físicas de la delimitación de los espacios, señalizaciones). Cada uno de entre ellos tiene un área de eficiencia, que puede definirse simplemente como el conjunto de actores para quiénes funciona como un recurso, una limitación y un reto. Por ejemplo, el área de eficiencia de un periódico puede ser definida por sus lectores, aquella de una ley por el conjunto de personas a las cuales se les aplica, la de una barrera que organiza una fila de espera en el aeropuerto por el conjunto de viajeros concernidos, etc. Estas amplitudes son evidentemente muy variables. Las mediaciones diádicas solo conciernen dos personas. Desde el momento en que ellas involucran al menos tres personas, uno se encuentra frente a un colectivo. Lo que distingue el colectivo de la red es la forma de compartir los recursos de mediación, 
que son específicos a cada relación en la red mientras que ellos son (al menos teóricamente) compartidos por el colectivo. Mientras que los recursos circulan a lo largo de los canales relacionales dentro de la red, ellos son accesibles según las reglas de acceso en un colectivo.

Una relación interpersonal es en consecuencia un conjunto de recursos de mediaciones diádicas (es decir específica para los protagonistas de la relación) que permite la coordinación entre los actores, la circulación o la transmisión de recursos.

\section{Referencias}

Bidart Claire y Lavenu Daniel (2005). "Evolutions of personal networks and life events", Social Networks, 27, $n^{\circ} 4$, pp. 359-376.

Chaulet Johann (2007), « La confiance médiatisée. La confiance et sa gestion au sein des communications médiatisées », thèse de sociologie, Université de Toulouse 2 Le Mirail.

Cochoy Franck (2002). Une Sociologie du Packaging ou l'âne de Buridan face au marché. París : PUF.

Cook Karen S. (dir.) (2001). Trust in society. New-York: Russel Sage Foundation.

Degenne Alain y Forsé Michel (1994). Les réseaux sociaux. París : Armand Colin.

Ferrand A., 2006, "Redes heterogéneas de discusión y pluralismo cognitivo.", REDES. Revista hispana para el análisis de redes sociales. Vol.10, art. 2, julio 2006. http://revista-redes. rediris.es/pdf-vol10/vol10_2.pdf

Fischer Claude S. (1982). To Dwell Among Friends. Chicago: University of Chicago Press.

Granovetter Mark S. (1973). "The strength of weak ties." American Journal Of Sociology, Vol. 78, pp.1360-1380.

Granovetter Mark S. (1974). Getting a job. Harvard: Harvard University Press

Granovetter Mark S. (1985). "Economic action and social structure : the problem of embeddedness". American J ournal of Sociology, Vol. 91, pp.481-510.

Grossetti Michel y Bès Marie-Pierre (2001). "Encastrements et découplages dans les relations science - industrie "., Revue Française de Sociologie, Vol. 42, $n^{\circ} 2$, pp.327-355.

Grossetti Michel (2004). Sociologie de l'imprévisible. Dynamiques de l'activité et des formes sociales. Paris : PUF.

Grossetti Michel (2005). "Where do social relations come from?: A study of personal networks in the Toulouse area of France", Social Networks, Vol. 27, pp.289-300.

Grossetti Michel (2007). "Are French networks different ? "Social Networks, Vol. 29, n³, pp. 391-404. 
Hennion Antoine (1993). La passion musicale. Une sociologie de la mediation. Paris: Metailié.

Mullins, Nicholas C. (1972). "The Development of a Scientific Speciality: the Phage Group and the Origins of Molecular Biology", Minerva, vol.19, p. 52-82.

Wellman Barry y Berkowitz Stanley D (1988). "Introduction: studying social structures" in Barry Wellman and S.D. Berkowitz (ed.), Social structures. A network approach, 1988, 1997, Greenwich USA and London GB: JAI Press, pp. 1-14

White Harrison C. (2002). Market from networks. Socioeconomic models of production, Princeton and Oxford: Princeton University Press,.

White Harrison C. (1995). "Passages réticulaires, acteurs et grammaire de la domination", Revue Française de Sociologie, Vol. 36, pp.705-723.

White. Harrison C. (1992). Identity and Control. A structural theory of social action. Princeton and Oxford: Princeton University Press. 